\title{
HEAT AND MASS TRANSFER EFFECTS ON MHD FLOW PAST AN INCLINED POROUS PLATE IN THE PRESENCE OF CHEMICAL REACTION
}

\author{
A. SANDHYA* \\ Research Scholar JNTUK Kakinada, AP-533003 \\ Department of Mathematics, VR Siddhartha Engineering College \\ Kanur, Vijayawada-520007, INDIA \\ E-mail: akuri.sandhya@gmail.com \\ G.V. RAMANA REDDY \\ Department of Mathematics, KLEF \\ Vaddeswaram, AP-522502, INDIA \\ E-mail: gvrr1976@gmail.com \\ G.V.S.R. DEEKSHITULU \\ Department of Mathematics, JNTUK Kakinada \\ AP-533003, INDIA \\ E-mail: deekshitulu_g@yahoo.com
}

\begin{abstract}
The impact of heat and mass transfer effects on an MHD flow past an inclined porous plate in the presence of a chemical reaction is investigated in this study. An effort has been made to explain the Soret effect and the influence of an angle of inclination on the flow field, in the presence of the heat source, chemical reaction and thermal radiation. The momentum, energy and concentration equations are derived as coupled second order partial differential equations. The model is non-dimensionalized and shown to be controlled by a number of dimensionless parameters. The resulting dimensionless partial differential equations can be solved by using a closed analytical method. Numerical results for pertaining parameters, such as the Soret number (Sr), Grashof number (Gr) for heat and mass transfer, the Schmidt number (Sc), Prandtl number (Pr), chemical reaction parameter $(\mathrm{Kr})$, permeability parameter $(\mathrm{K})$, magnetic parameter $(\mathrm{M})$, skin friction $(\tau)$, Nusselt number $(\mathrm{Nu})$ and Sherwood number $(\mathrm{Sh})$ on the velocity, temperature and concentration profiles are presented graphically and discussed qualitatively.
\end{abstract}

Keywords: chemical reaction, heat source, inclined plate, porous medium, thermal radiation.

\section{Introduction}

MHD free convection heat and mass transfer flows through a porous medium have always being a topic of interest due to their abundance in nature and varied applications in many branches of science and technology. The applications are many, for instance fibre and granular insulation geothermal systems in the heating and cooling chambers, fossils fuel combustion energy process and astrophysical flows, etc. The role of MHD free convection flows through porous media is very significant, particularly in the field of petroleum, technology for the flow of oil through porous rocks, and in chemical engineering for purification and filtration process, and so on. Fujji and Imura [1] described on experimental study concerning natural convection heat transfer from a plate with arbitrary inclination. Lin et al. [2] studied the new formulation for

\footnotetext{
${ }^{*}$ To whom correspondence should be addressed
} 
laminar free convection from an arbitrarily inclined isothermal plate for fluids of any Prandtl number ( $\operatorname{Pr}$ ) between 0.001 and $\infty$. Helmy [3] presented an unsteady two-dimensional laminar free convection flow of an incompressible, electrical conducting fluid past an infinite vertical plate in a porous medium by assuming the plate temperature to be oscillating with time about a constant nonzero value. Mhone et al. [4] studied the effect of radiation on an unsteady MHD flow through a channel field with a saturated porous medium. Kim et al. [5] presented an exact analysis of radiation convective flow heat and mass transfer over an inclined plate in a porous medium. The effect of chemical reaction on an MHD flow of a viscoelastic fluid through a porous medium was studied by Nayak et al. [6]. Reddy et al. [7] presented the effect of the critical parameters on an MHD flow in a porous channel when the suction decreases exponentially. The effect of mass transfer on a free convection flow past a vertical isothermal plate was examined by Gebhart and Para [8]. Decay et al. [9] studied combined effects of thermal radiation and chemical reaction on a free convection flow past a vertical plate in a porous medium. Mass transfer effects on an MHD mixed convective flow from a vertical surface with Ohmic heating and viscous dissipation were studied by Sri Hari Babu and Ramana Reddy [10]. Barik et al. [11] studied thermal radiation effects on the unsteady MHD flow past an inclined plate in the presence of chemical reaction and viscous dissipation. The influence of the Soret number on an unsteady magneto hydro dynamic fluid flow in a past a semi-infinite vertical plate in the presence of viscous dissipation was discussed by Sheri and Raju et al. [12]. Srinivasa Raju et al. [13] studied both the analytical and numerical results of an unsteady MHD free convective flow past an exponentially moving vertical plate with heat absorption and chemical reaction. Aishah et al. [14] studied the heat and mass transfer in an unsteady hydro magnetic free convective flow through a porous medium past a positive vertical plate with uniform surface heat flux. Radiation and chemical reaction effects on an MHD flow along a moving vertical porous plate were studied Gorla et al. [15]. Manga Thai et al. [16] presented the MHD free convective flow past a vertical porous plate in the presence of radiation and heat generation. The problem of the MHD free convective flow with parabolic starting motion of an inclined vertical plate finds applications in steel industry, solar energy collection systems, aerospace and also in the design of high temperature chemical process systems. Sri Devi et al. [17] studied the Soret and Dufour effects on the MHD flow with heat and mass transfer past a permeable stretching sheet in the presence of thermal radiation. Reddy et al. [18] discussed similarity transformations of heat and mass transfer effects on a steady MHD free convection dissipative fluid flow past an inclined porous surface with chemical reaction.

But in the above mentioned works dealing with parabolic starting motion of an infinite plate, the plate is considered to be vertical. So the present work aims to study the influence of an angle of inclination and the thermal diffusion of flow past an inclined plate moving with variable velocity in the presence of thermal radiation, heat source, and chemical reaction. Effects of the variations in the plate velocity, temperature, and concentration are assumed to be time-dependent. Such an assumption is common in practice. The mass transfer effects occurring in numerous physical situations that involve diffusive and convective transport of chemical species within physical systems are taken into account. Mass transferrelated problems are also encountered in chemical engineering for separation processes, removal of pollutants in many chemical reactions, and so on. The governing equations are linear and an exact solution to the problem has been obtained by applying the closed analytical method. The work focuses on a few physical situations, particularly in seepage flow, in which the flow domain is not necessarily vertical or horizontal. Such a model may have industrial and engineering applications as the vertical angle of inclination of the plate serves in reducing the fluid velocity. It needs to be mentioned that in the reported literature no work has been done on flow past an inclined plate moving with parabolic velocity with respect to time.

\section{Mathematical formulation}

Consider an unsteady MHD free convective flow of a viscous incompressible and electrically conducting fluid past an infinite inclined porous plate with time-dependent variable plate velocity, heat and mass transfer in a saturated porous medium. The $x^{*}$-axis is taken along the leading edge of an inclined plate with an angle of inclination $\alpha$ to the vertical direction. The $y^{*}$ - axis is taken normal to the plate. Initially, both 
the fluid and the plate were at rest with constant temperature $T_{\infty}^{*}$ and constant concentration $C_{\infty}^{*}$. At time $t^{*}>$ 0 , the plate starts moving with a velocity $u^{*}=u_{0}\left(t^{*} / t_{0}\right)^{2}$ in its own plane against the gravitational field.

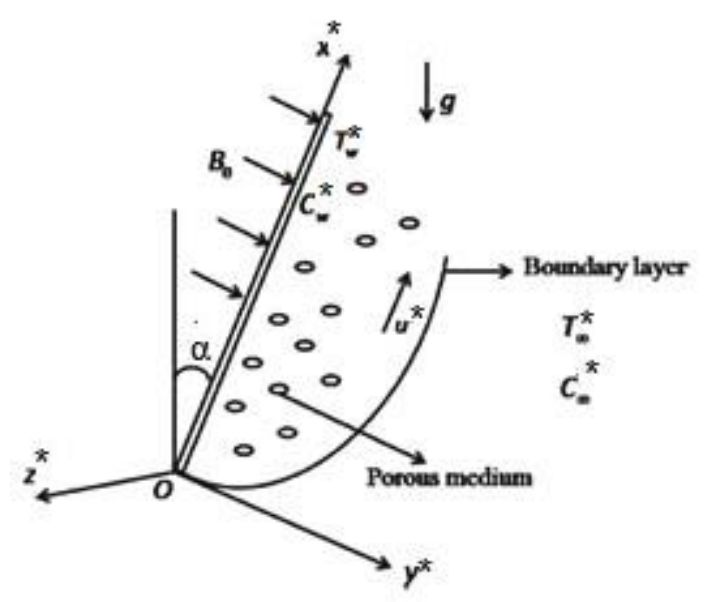

Fig.1. Physical configuration of the problem.

The plate temperature and mass diffusion from the plate into the fluid are increased linearly with reference to time. A uniform magnetic field of strength $B_{0}$ is applied transversely to the plate along the $y^{*}-$ direction. The Reynolds number is assumed to be very small which corresponds to a negligible induced magnetic field when compared to the externally applied force, and hence $\boldsymbol{B}=\left(0, B_{0}, 0\right)$ is the total magnetic field acting on the fluid. Further, it is considered that the viscous dissipation of energy is negligible and that the fluid is an optically thin gray radiating but non scattering medium. All the fluid properties are supposed to be of a fixed value except the density in the buoyancy force term.

In view of the above assumptions, the usual Boussinesq approximation, the governing equations considered are as follows:

Momentum equation

$$
\frac{\partial u^{*}}{\partial t^{*}}=g\left[\beta\left(T^{*}-T_{\infty}^{*}\right) \cos \alpha+\beta^{*}\left(C^{*}-C_{\infty}^{*}\right) \cos \alpha\right]+v \frac{\partial^{2} u^{*}}{\partial y^{* 2}}-\frac{\sigma B_{0}^{2}}{\rho} u^{*}-\frac{v u^{*}}{k_{p}^{*}} .
$$

Energy equation

$$
\rho C_{p} \frac{\partial T^{*}}{\partial t^{*}}=K_{T} \frac{\partial^{2} T^{*}}{\partial y^{* 2}}-\frac{\partial q_{r}}{\partial y^{*}}+Q^{*}\left(T^{*}-T_{\infty}^{*}\right) .
$$

Species equation

$$
\frac{\partial C^{*}}{\partial t^{*}}=D_{M} \frac{\partial^{2} C^{*}}{\partial y^{* 2}}-K_{C}^{*}\left(C^{*}-C_{\infty}^{*}\right)+D_{T} \frac{\partial^{2} T^{*}}{\partial y^{* 2}}
$$

where $u^{*}$ is the component of velocity along the $x^{*}$ - axis, $v$ is the kinematic viscosity, $g$ is the acceleration due to gravity, $\beta$ is the volume expansion coefficient for the heat transfer and $\beta^{*}$ is the volume expansion coefficient for the mass transfer, $k_{p}^{*}$ is the permeability of the porous medium, $q_{r}$ is the radiative heat flux, $\infty$ is the angle of inclination, $\sigma$ is the electrical conductivity of the fluid, $T^{*}$ is the fluid temperature, $T_{\infty}^{*}$ is the far field temperature, $K_{T}$ is the thermal conductivity, $K_{C}^{*}$ is the chemical reaction parameter, $Q^{*}$ is the 
heat source parameter, $\rho$ is the density of the fluid, $C_{P}$ is specific heat at constant pressure,$C^{*}$ is the species concentration, $C_{\infty}^{*}$ is the far field concentration, $D_{M}$ is the coefficient of molecular diffusivity, $D_{T}$ is the coefficient of thermal diffusion.

The initial and boundary conditions of the physical model are presented by

$$
\begin{aligned}
& t^{*} \leq 0: u^{*}=0, \quad T^{*}=T_{\infty}^{*}, \quad C^{*}=C_{\infty}^{*} \forall y^{*}, \\
& t^{*}>0: u^{*}=u_{0}\left(\frac{t^{*}}{t_{0}}\right)^{2}, \quad T^{*}=T_{\infty}^{*}+\frac{T_{\omega}^{*}-T_{\infty}^{*}}{v} u_{0}^{2}\left(\frac{t^{*}}{t_{0}}\right), \\
& C^{*}=C_{\infty}^{*}+\frac{C_{\omega}^{*}-C_{\infty}^{*}}{v} u_{0}^{2}\left(\frac{t^{*}}{t_{0}}\right) \quad \text { at } \quad y^{*}=0, \\
& u^{*} \rightarrow 0, \quad T^{*} \rightarrow T_{\infty}^{*}, \quad C^{*} \rightarrow C_{\infty}^{*} \quad \text { as } \quad y^{*} \rightarrow \infty .
\end{aligned}
$$

Using the Rosseland approximation the rate of radioactive heat flux is obtained by

$$
\frac{\partial q_{r}}{\partial y^{*}}=-4 a^{*} \sigma^{*}\left(T_{\infty}^{* 4}-T^{* 4}\right)
$$

Let us assume that the temperature differences are sufficiently small within the flow such that $T^{* 4}$ can be represented as a linear function of the temperature. This is accomplished by expanding $T^{* 4}$ in a Taylor series about $T_{\infty}{ }^{* 4}$ and neglecting the higher-order terms. We will get

$$
T^{* 4} \cong 4 T_{\infty}^{* 3} T^{*}-3 T_{\infty}^{* 4} \text {. }
$$

Using Eqs (2.5) and (2.6) in Eq.(2.2), we obtain

$$
\rho C_{p} \frac{\partial T^{*}}{\partial t^{*}}=K_{T} \frac{\partial^{2} T^{*}}{\partial y^{* 2}}-16 a^{*} \sigma^{*} T_{\infty}^{* 3}\left(T^{*}-T_{\infty}^{*}\right)+Q^{*}\left(T^{*}-T_{\infty}^{*}\right) .
$$

Non-dimensional quantities are defined by

$$
\begin{aligned}
& y=\frac{y^{*} u_{0}}{v}, \quad u=\frac{u^{*}}{u_{0}}, \quad t=\frac{t^{*}}{t_{0}}, \quad \theta=\frac{T^{*}-T_{\infty}^{*}}{T_{\omega}^{*}-T_{\infty}^{*}}, \quad \phi=\frac{C^{*}-C_{\infty}^{*}}{C_{\omega}^{*}-C_{\infty}^{*}}, \quad \operatorname{Gr}=\frac{g \beta v\left(T_{\omega}^{*}-T_{\infty}^{*}\right)}{u_{0}^{3}}, \\
& \mathrm{Gm}=\frac{g \beta^{*} v\left(C_{\omega}^{*}-C_{\infty}^{*}\right)}{u_{0}^{3}}, \quad \operatorname{Pr}=\frac{\mu C_{p}}{K_{T}}, \quad M=\frac{\sigma B_{0}^{2} v}{\rho u_{0}^{2}}, \quad K_{p}=\frac{u_{0}^{2} k_{p}^{*}}{v^{2}}, \quad K_{r}=\frac{v k_{c}^{*}}{u_{0}^{2}}, \\
& R=\frac{16 a^{*} v^{2} \sigma^{*} T_{\infty}^{*}}{K_{T} u_{0}^{2}}, \quad Q=\frac{Q^{*} v}{\rho C_{p} u_{0}^{2}}, \quad \mathrm{Sc}=\frac{v}{D_{M}}, \quad \mathrm{Sr}=\frac{D_{T}}{v} \frac{T_{\omega}^{*}-T_{\infty}^{*}}{C_{\omega}^{*}-C_{\infty}^{*}} .
\end{aligned}
$$


The equations in non-dimensional form are

$$
\begin{aligned}
& \frac{\partial u}{\partial t}=\frac{\partial^{2} u}{\partial y^{2}}-M u-\frac{u}{K_{p}}+\mathrm{Gr} \theta \cos \alpha+\mathrm{Gm} \phi \cos \alpha, \\
& \frac{\partial \theta}{\partial t}=\frac{1}{\operatorname{Pr}} \frac{\partial^{2} \theta}{\partial y^{2}}-\frac{R}{\operatorname{Pr}} \theta+Q \theta \\
& \frac{\partial \phi}{\partial t}=\frac{1}{\operatorname{Sc}} \frac{\partial^{2} \phi}{\partial y^{2}}-K_{r} \phi+\operatorname{Sr} \frac{\partial^{2} \theta}{\partial y^{2}}
\end{aligned}
$$

The corresponding initial and boundary conditions are

$$
\begin{aligned}
& t \leq 0: \quad u=0, \quad \theta=0, \quad \phi=0 \forall y, \\
& t>0: \quad u=t^{2}, \quad \theta=t, \quad \phi=t \quad \text { at } \quad y=0, \\
& u \rightarrow 0, \quad \theta \rightarrow 0, \quad \phi \rightarrow 0 \quad \text { as } \quad y \rightarrow \infty .
\end{aligned}
$$

\section{Method of solution}

Equations (2.9)-(2.11) are coupled non-linear partial differential equations and these equations can be solved by a closed form method, i.e. the equations can be reduced to a set of ordinary differential equations which can be solved analytically. This can be done by representing the velocity, temperature and concentration of the fluid in the neighbourhood of the plate as

$$
\begin{aligned}
& u(y, t)=u_{0}(y) e^{i \omega t}, \\
& \theta(y, t)=\theta_{0}(y) e^{i \omega t}, \\
& \phi(y, t)=\phi_{0}(y) e^{i \omega t} .
\end{aligned}
$$

Substituting (3.1) in Eqs (2.9) -(2.11) and (2.12) we get the differential equations

$$
\begin{aligned}
& u_{0}^{\prime \prime}-k_{3}^{2} u_{0}=-\left[\mathrm{Gr} \theta_{0} \cos \alpha+\mathrm{Gm} \phi_{0} \cos \alpha\right], \\
& \theta_{0}^{\prime \prime}-k_{1}^{2} \theta_{0}=0 . \\
& \phi_{0}^{\prime \prime}-k_{1}^{2} \phi_{0}=-\operatorname{SrSc} \theta_{0}^{\prime \prime} .
\end{aligned}
$$

The corresponding boundary conditions are 


$$
\begin{array}{ll}
t \leq 0: \quad u_{0}=0, \quad \theta_{0}=0, \quad \phi_{0}=0 \forall y, \\
t>0: \quad u_{0}=t^{2} e^{-i \omega t}, \quad \theta_{0}=t e^{-i \omega t}, \quad \phi_{0}=t e^{-i \omega t} \quad \text { at } \quad y=0, \\
\mathrm{u}_{0} \rightarrow 0, \quad \theta_{0} \rightarrow 0, \quad \phi_{0} \rightarrow 0 \quad \text { as } \quad y \rightarrow \infty
\end{array}
$$

solving Eqs (3.2)-(3.4) and using Eq.(3.5) the velocity, temperature and concentration fields are as follows.

$$
\begin{aligned}
& \theta(y, t)=t e^{-k_{1} y}, \\
& \phi(y, t)=A_{2} e^{-k_{2} y}+A_{1} e^{-k_{1} y}, \\
& u(y, t)=A_{7} e^{-k_{3} y}+A_{6} e^{-k_{1} y}+A_{4} e^{-k_{2} y} .
\end{aligned}
$$

The skin friction, Nusselt number and Sherwood number are significant physical parameters for this type of boundary layer flow.

\section{Skin friction}

Knowing the velocity field, the skin friction on the plate $y=0$ in non-dimensional form is given by

$$
\begin{aligned}
& \tau=-\left.\frac{\partial u}{\partial y}\right|_{y=0}, \\
& \tau=\left[k_{3} A_{7}+k_{1} A_{6}+k_{2} A_{4}\right] .
\end{aligned}
$$

\section{Nusselt number}

Knowing the temperature field, the rate of heat transfer coefficients can be obtained which is in nondimensional form in terms of the Nusselt number and is given by

$$
\begin{aligned}
& \mathrm{Nu}=-\left.\frac{\partial \theta}{\partial y}\right|_{y=0}, \\
& \mathrm{Nu}=t k_{1} .
\end{aligned}
$$

\section{Sherwood number}

The rate of the mass transfer at that plate in terms of the Sherwood number is given by

$$
\begin{aligned}
& \mathrm{Sh}=-\left.\frac{\partial \phi}{\partial y}\right|_{y=0}, \\
& \mathrm{Sh}=\left(t-A_{1}\right) k_{2}+k_{1} A_{1} .
\end{aligned}
$$




\section{Results and discussion}

Figure 2 demonstrates the effect of the magnetic field on the velocity profiles. It is observed that the velocity decreases with an increasing magnetic parameter $(M)$. This is due to the Lorentz force acting on the fluid flow. This type of resisting force slows down the fluid velocity as shown in this figure. The effect of the permeability parameter in the velocity profile is depicted in Fig.3. It is observed that the velocity increases with an increasing permeability parameter. For the case of different values of the thermal Grashof number (Gr), the velocity profiles on the boundary layer are shown in Fig.4. As expected, it is observed that an increase in the Grashof number ( $\mathrm{Gr}$ ) leads to an increases in the values of velocity due to enhancement in the buoyancy force. Here the positive values of the Grashof number (Gr) represent the surface cooling. Also, as the Grashof number increases, the peak values of the velocity increase rapidly near the porous plate and then decay smoothly to the free stream velocity. The influence of the modified Grashof number $(\mathrm{Gm})$ on the velocity profiles is shown in Fig.5. It is observed that as the modified Grashof number increases, the velocity profiles increase. Figures 6 and 7 represent the velocity and temperature profiles for different values of the Prandtl number (Pr). It is observed that the Prandtl number (Pr) increases the velocity and the temperature profiles decrease due to the thermal boundary layer thickness. Because of the smaller values of the Prandtl numbers, the rate of heat transfer is reduced and the boundary layer is thicker. Figures 8 and 9 illustrate the influence of the radiation parameter $(R)$ on the velocity and temperature profiles. It is observed that as the thermal radiation increases, the velocity and temperature decrease due to the boundary layer getting thicker. Figures 10 and 11 illustrate the effect of the heat source $(Q)$ on the velocity and temperature fields. It is visible that for the increase of the heat source parameter $(Q)$, the velocity and temperatures are both decreasing. Effects of chemical reaction parameters and the velocity and concentration profiles are presented in Figs 12 and 13, respectively. It is observed that the velocity and concentration profiles are decaying functions of amassed values of the chemical reaction parameter $(\mathrm{Kr})$. For the increased values of the chemical reaction parameter both velocity and concentration profiles decrease since the molecular diffusivity diminishes. Figures 14 and 15 illustrate the effect of the Schmidt number (Sc) on the velocity and concentration profiles. It is observed that as the Schmidt number decreases both velocity and concentration profiles also decreases, due to the molecular diffusivity and boundary layer thickness. On the other hand, the effects of the Soret number ( $\mathrm{Sr}$ ) on velocity and concentration profiles are completely opposite to that of the Schmidt number (Sc) as clearly observed in Figs 16 and 17. Figure 18 represents the velocity profile for different values of the angle of inclination $(\alpha)$. It is observed that as the angle of inclination $(\alpha)$ increases, the velocity profile decreases.

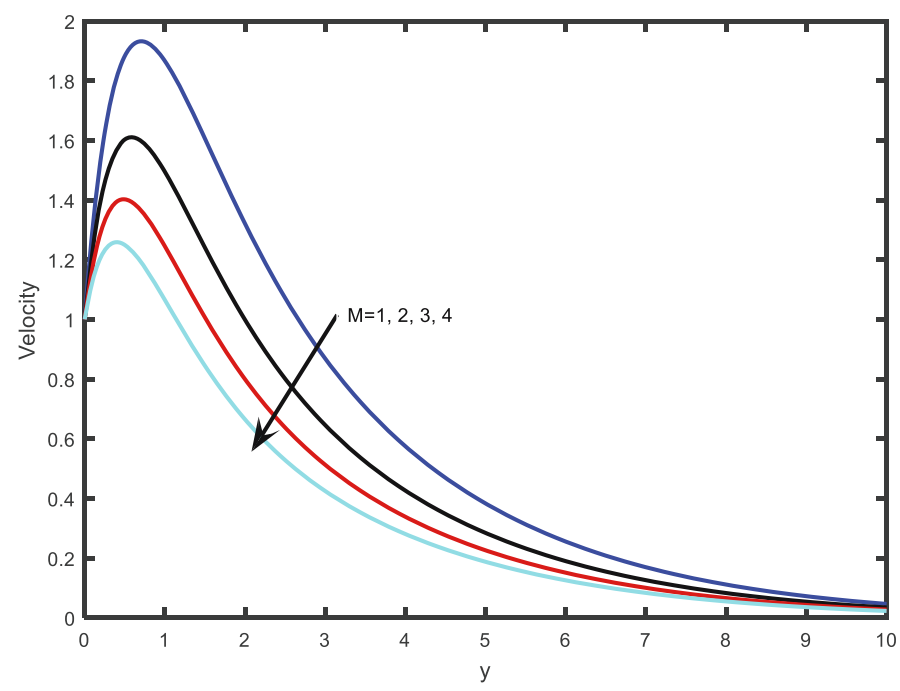

Fig.2. Velocity profiles for different values of the magnetic parameter $(M)$. 


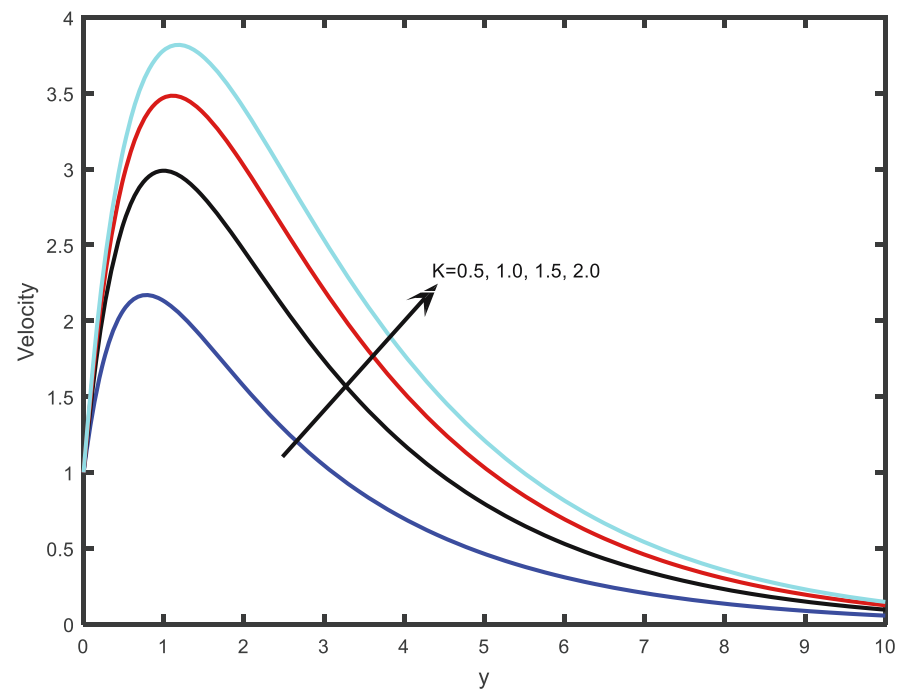

Fig.3. Velocity profiles for different values of the permeability parameter $(K)$.

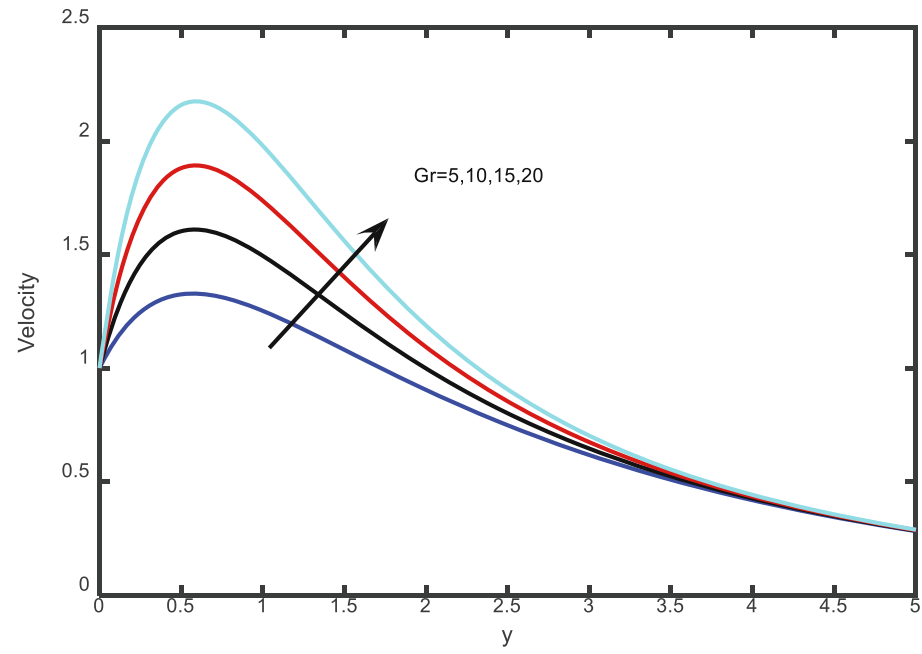

Fig.4. Velocity profiles for different values of the Grashof number (Gr).

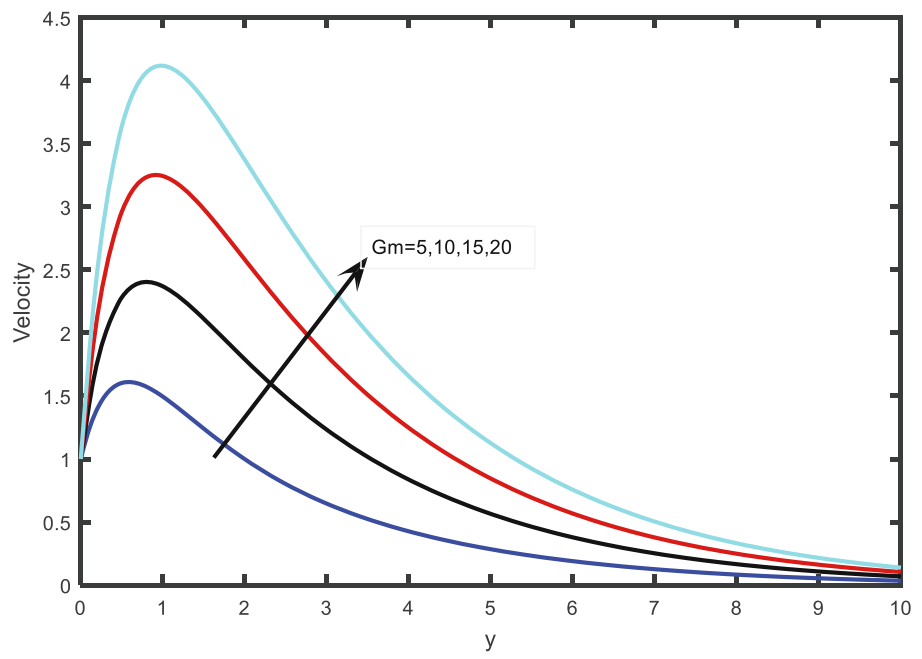

Fig.5. Velocity profiles for different values of the modified Grashof number (Gm). 


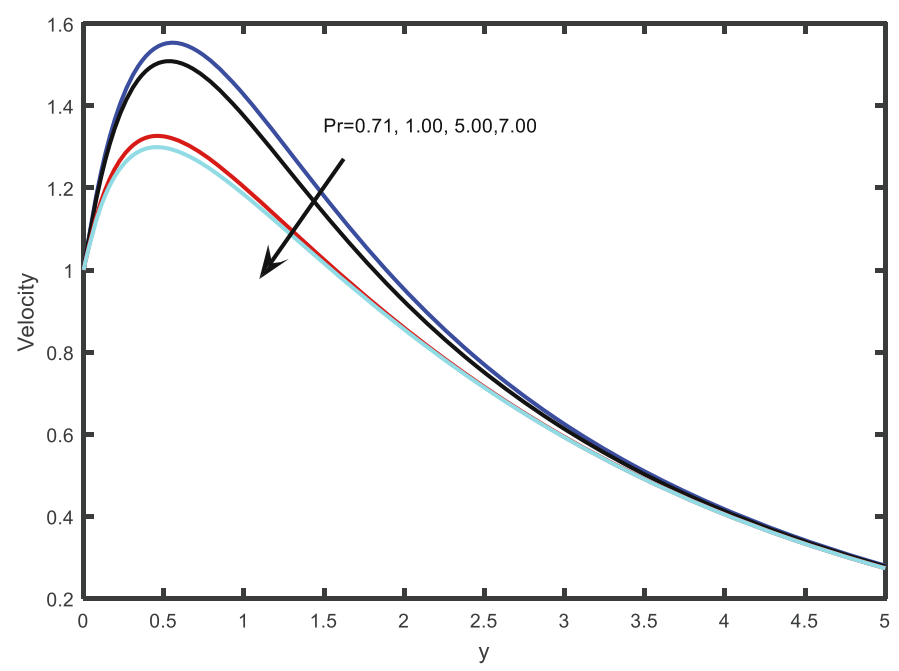

Fig.6. Velocity profiles for different values of the Prandtl number (Pr).

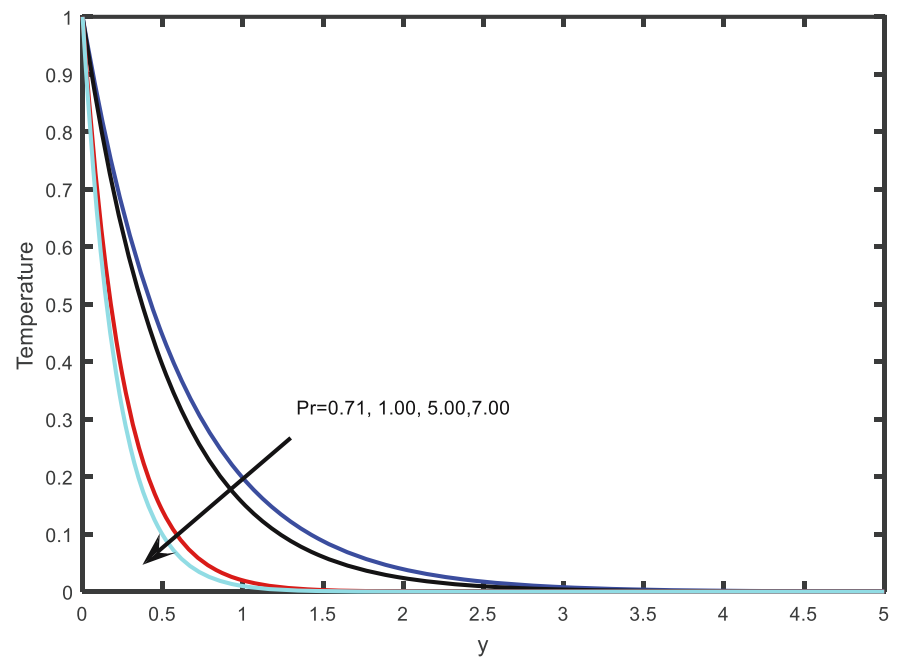

Fig.7. Temperature profiles for different values of the Prandtl number (Pr).

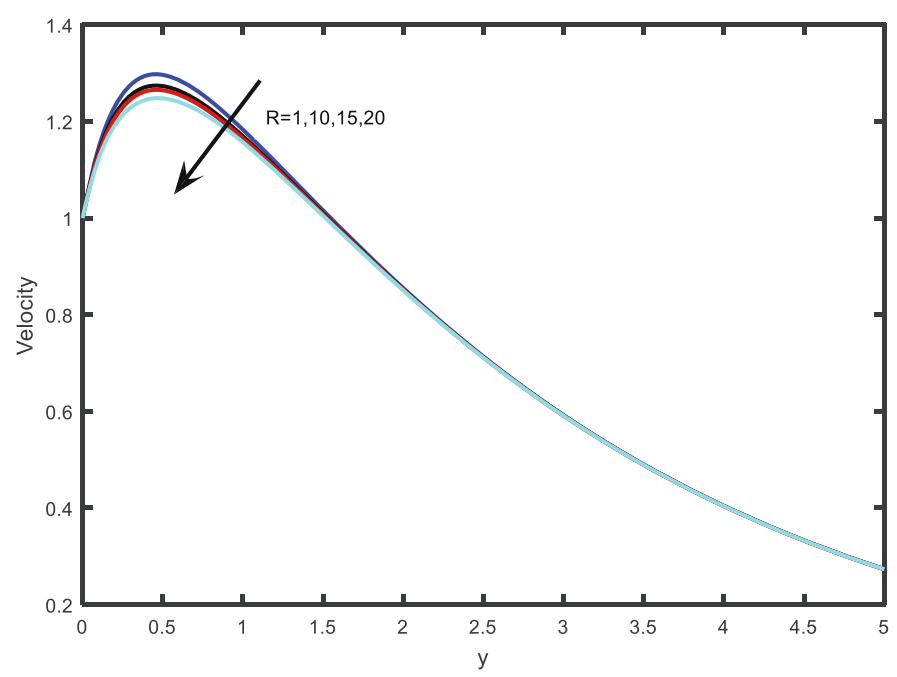

Fig.8. Velocity profiles for different values of the radiation parameter $(R)$. 


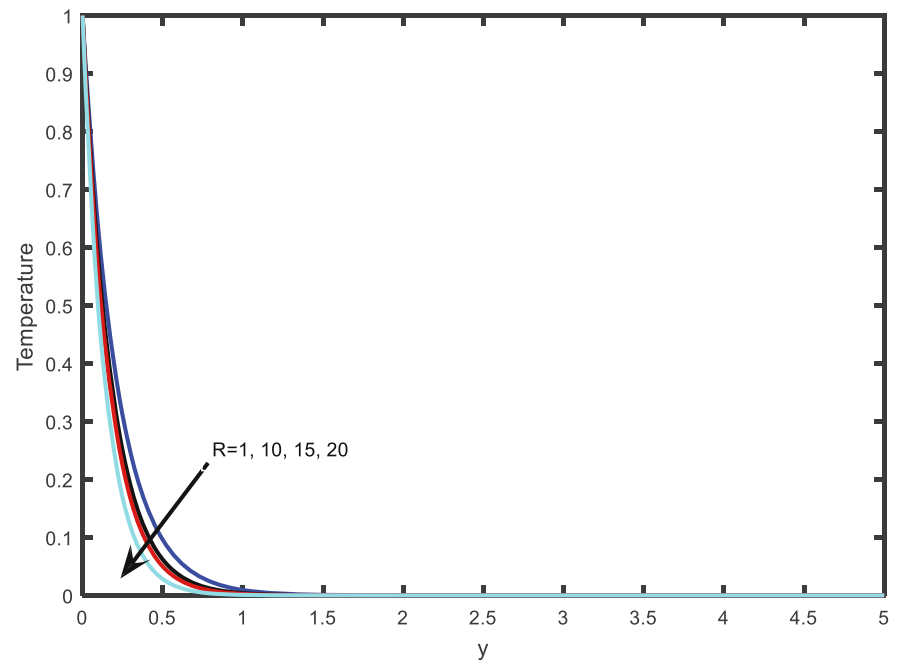

Fig.9. Temperature profiles for different values of the radiation parameter $(R)$.

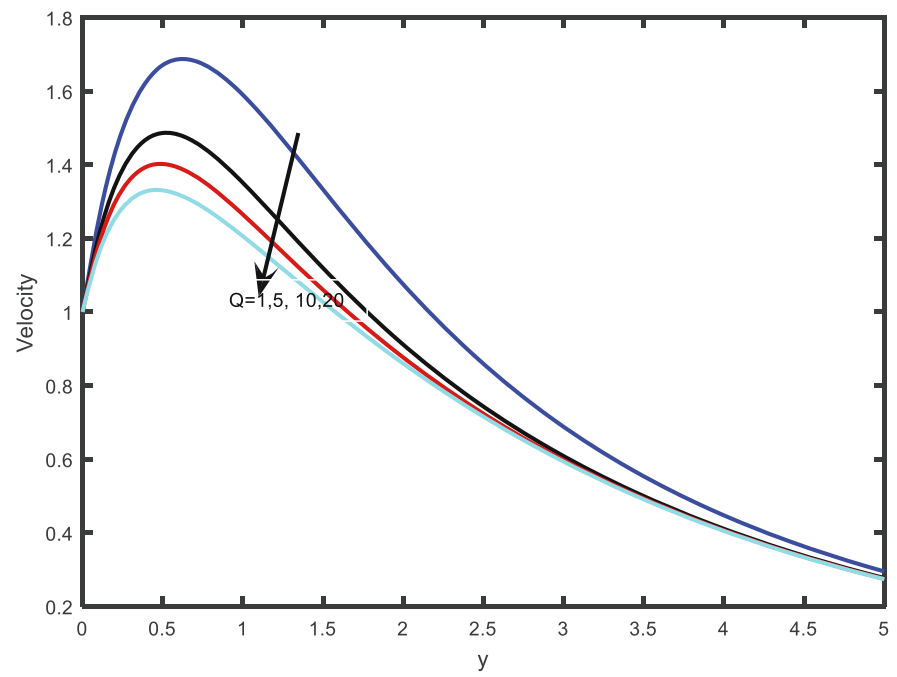

Fig.10. Velocity profiles for different values of the heat source $(Q)$.

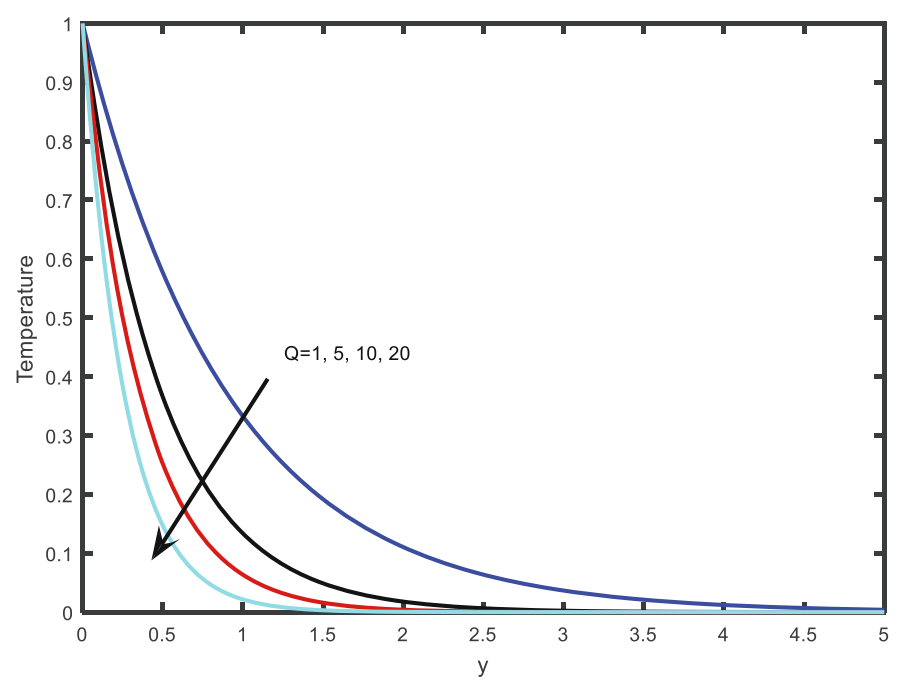

Fig.11. Temperature profiles for different values of the heat source $(Q)$. 


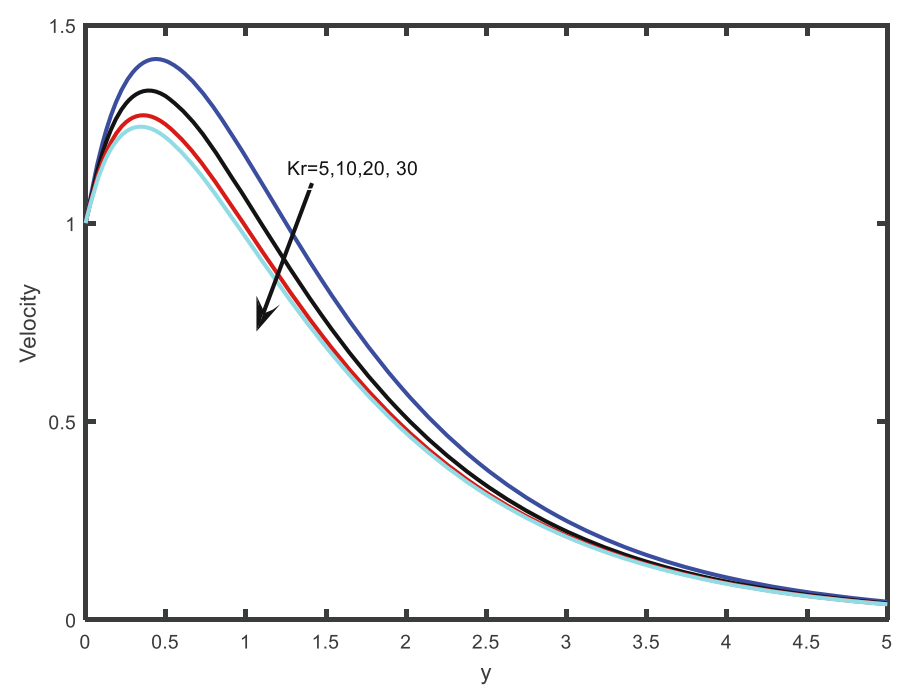

Fig.12. Velocity profiles for different values of the chemical reaction parameter $(\mathrm{Kr})$.

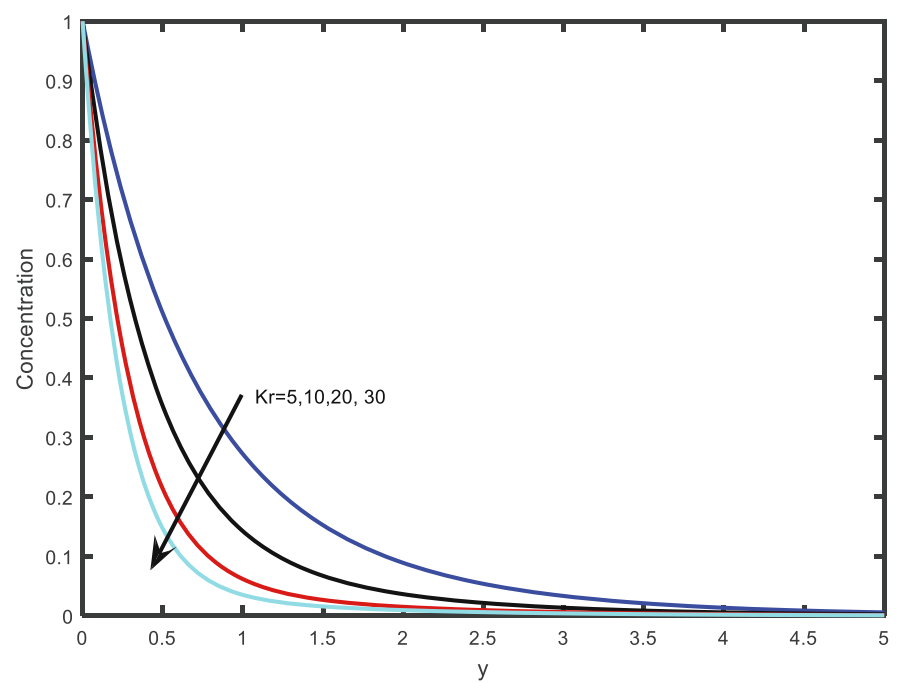

Fig.13. Concentration profiles for different values of the chemical reaction parameter $(\mathrm{Kr})$.

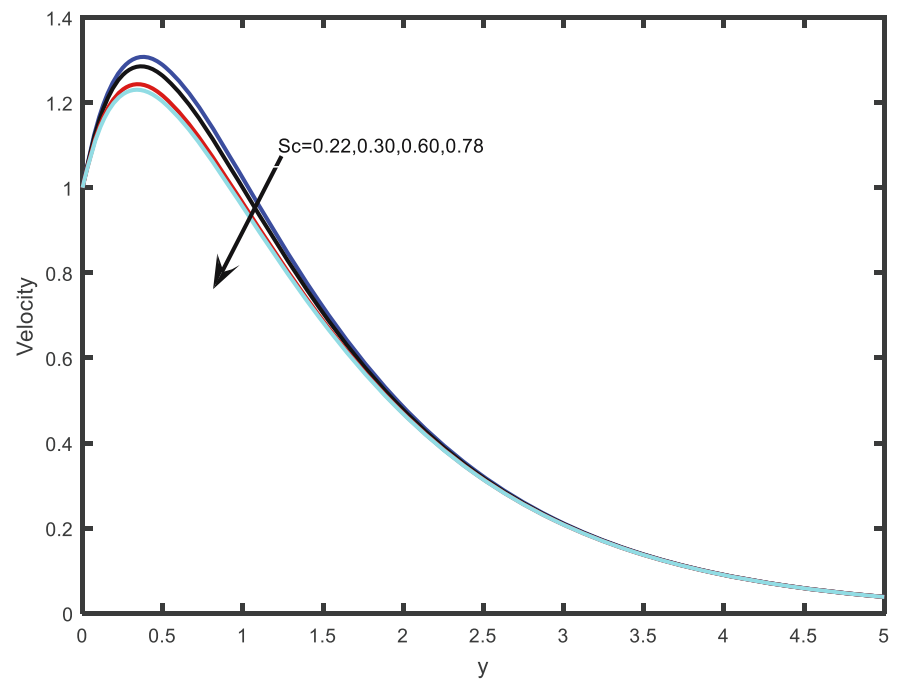

Fig.14. Velocity profiles for different values of the Schmidt number (Sc). 


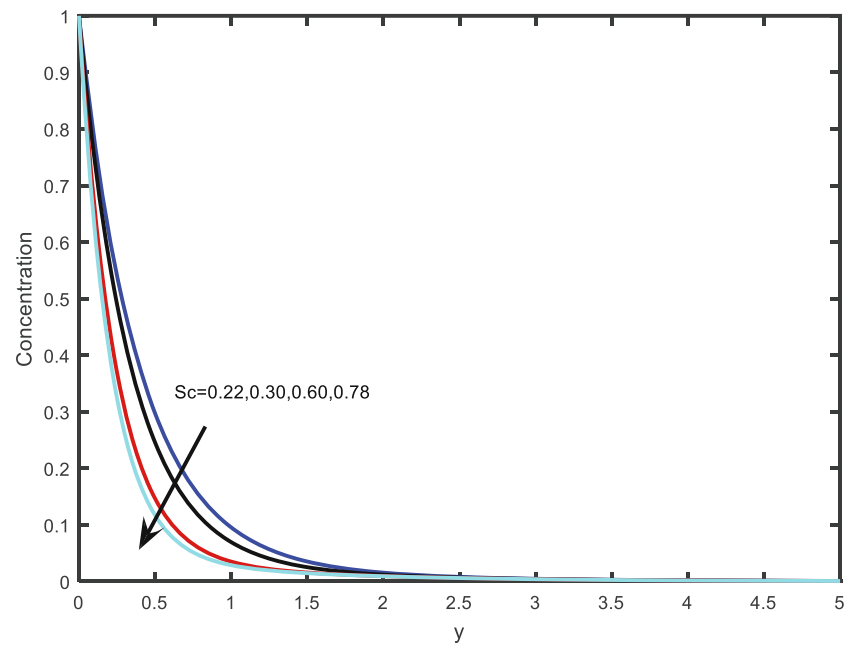

Fig.15. Concentration profiles for different values of the Schmidt number (Sc).

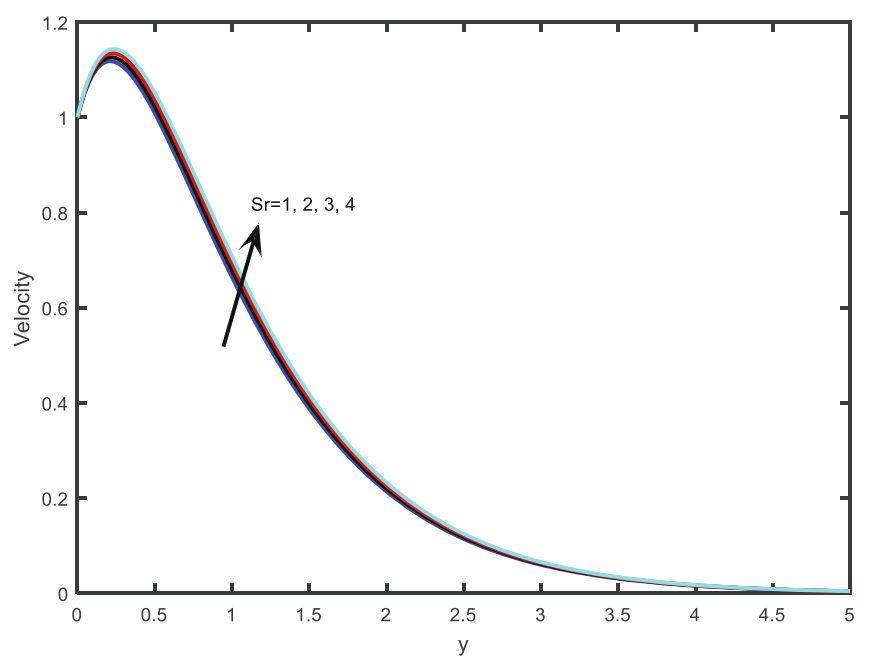

Fig.16.Velocity profiles for different values of the Soret number (Sr).

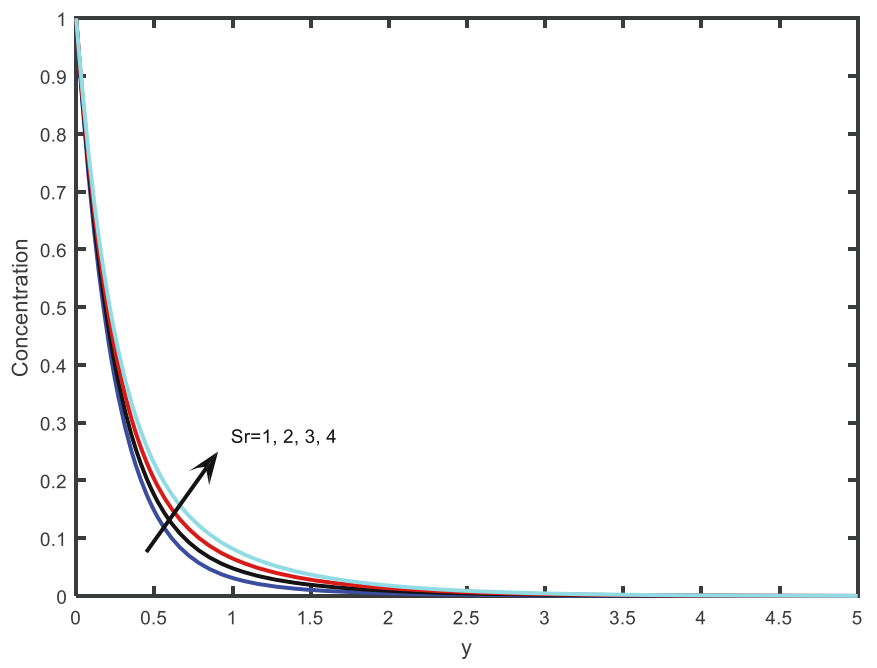

Fig.17. Concentration profiles for different values of the Soret number ( $\mathrm{Sr})$. 


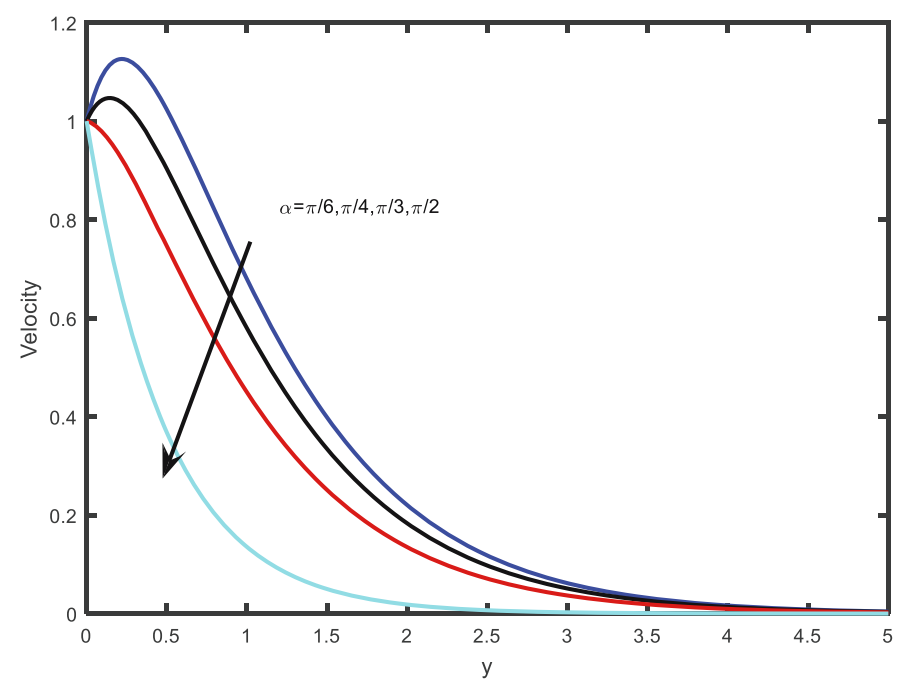

Fig.18. Velocity profiles for different values of the angle of inclination $(\alpha)$.

Table 1. Numerical results.

\begin{tabular}{|c|c|c|c|c|c|c|c|c|c|c|c|c|c|c|c|}
\hline $\mathbf{G r}$ & Gm & Sc & $\mathrm{Sr}$ & $\boldsymbol{R}$ & $Q$ & $\alpha$ & $T$ & $\omega$ & $K$ & Pr & $\mathbf{K r}$ & $M$ & $\tau$ & $\mathrm{Nu}$ & Sh \\
\hline 5 & 5 & 0.6 & 2 & 0.5 & 2 & 30 & 1 & 0.1 & 0.5 & 0.71 & 0.5 & 1 & 1.0473 & 0.0370 & 0.9595 \\
\hline 10 & 5 & 0.6 & 2 & 0.5 & 2 & 30 & 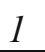 & 0.1 & 0.5 & 0.71 & 0.5 & 1 & 0.7151 & .0370 & 9595 \\
\hline 15 & 5 & 0.6 & 2 & 0.5 & 2 & 30 & 1 & 0.1 & 0.5 & 0.71 & 0.5 & 1 & 3829 & 0370 & 9595 \\
\hline 20 & 5 & 0.6 & 2 & 0. & 2 & 30 & 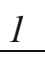 & 0.1 & 0.5 & 0.71 & 0.5 & 1 & 0.0507 & 0370 & 9595 \\
\hline 25 & 5 & 0.6 & 2 & 0.5 & 2 & 30 & 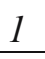 & 0.1 & 0.5 & 0.71 & 0.5 & 1 & -0.2814 & 0.0370 & 0.9595 \\
\hline 5 & 5 & 0.6 & 2 & 0.5 & 2 & 30 & 1 & 0.1 & 0.5 & 0.71 & 0.5 & 1 & 1.0473 & 0.0370 & 9595 \\
\hline 5 & 10 & 0.6 & 2 & 0.5 & 2 & 30 & 1 & 0.1 & 0.5 & 0.71 & 0.5 & 1 & 0.6944 & 0.0370 & 595 \\
\hline 5 & 15 & 0.6 & 2 & 0.5 & 2 & 30 & 1 & 0.1 & 0.5 & 0.71 & 0.5 & 1 & 0.3416 & 0.0370 & 0.9595 \\
\hline 5 & 20 & 0.6 & 2 & 0.5 & 2 & 30 & 1 & 0.1 & 0.5 & 0.71 & 0.5 & 1 & -0.0113 & 0.0370 & 0.9595 \\
\hline 5 & 25 & 0.6 & 2 & 0.5 & 2 & 30 & 1 & 0.1 & 0.5 & 0.71 & 0.5 & 1 & -0.3641 & 0.0370 & 0.9595 \\
\hline 5 & 5 & 0.2 & 2 & 0.5 & 2 & 30 & 1 & 0.1 & 0.5 & 0.71 & 0.5 & 1 & 1.0067 & & \\
\hline 5 & 5 & 0.4 & 2 & 0.5 & 2 & 30 & 1 & 0.1 & 0.5 & 0.71 & 0.5 & 1 & 1.0276 & 0.0370 & 0.6911 \\
\hline 5 & 5 & 0.6 & 2 & 0.5 & 2 & 30 & 1 & 0.1 & 0.5 & 0.71 & 0.5 & 1 & 1.0473 & 0.0370 & 0.9595 \\
\hline 5 & 5 & 0.8 & 2 & 0.5 & 2 & 30 & 1 & 0.1 & 0.5 & 0.71 & 0.5 & 1 & 1.0664 & 0.0370 & 1.2187 \\
\hline 5 & 5 & 1 & 2 & 0.5 & 2 & 30 & 1 & 0.1 & 0.5 & 0.71 & 0.5 & 1 & 1.0850 & 0.0370 & 1.4693 \\
\hline 5 & 5 & 0.6 & 0 & 0.5 & 2 & 30 & 1 & 0.1 & 0.5 & 0.71 & 0.5 & 1 & 1.0627 & 0.0370 & 0.5504 \\
\hline 5 & 5 & 0.6 & 0.5 & 0.5 & 2 & 30 & 1 & 0.1 & 0.5 & 0.71 & 0.5 & 1 & 1.0588 & 0.0370 & 0.6527 \\
\hline 5 & 5 & 0.6 & 1 & 0.5 & 2 & 30 & 1 & 0.1 & 0.5 & 0.71 & 0.5 & 1 & 1.0550 & 0.0370 & 0.7550 \\
\hline 5 & 5 & 0.6 & 1.5 & 0.5 & 2 & 30 & 1 & 0.1 & 0.5 & 0.71 & 0.5 & 1 & 1.0511 & 0.0370 & 0.8572 \\
\hline 5 & 5 & 0.6 & 2 & 0.5 & 2 & 30 & 1 & 0.1 & 0.5 & 0.71 & 0.5 & 1 & 1.0473 & 0.0370 & 0.9595 \\
\hline 5 & 5 & 0.6 & 2 & 0.1 & 2 & 30 & 1 & 0.1 & 0.5 & 0.71 & 0.5 & 1 & 1.0495 & 0.0309 & 1.0083 \\
\hline 5 & 5 & 0.6 & 2 & 0.2 & 2 & 30 & 1 & 0.1 & 0.5 & 0.71 & 0.5 & 1 & 1.0493 & 0.0321 & 0.9983 \\
\hline 5 & 5 & 0.6 & 2 & 0.3 & 2 & 30 & 1 & 0.1 & 0.5 & 0.71 & 0.5 & 1 & 1.0489 & 0.0335 & 0.9870 \\
\hline
\end{tabular}




\begin{tabular}{|c|c|c|c|c|c|c|c|c|c|c|c|c|c|c|c|}
\hline 5 & 5 & 0.6 & 2 & 0.4 & 2 & 30 & 1 & 0.1 & 0.5 & 0.71 & 0.5 & 1 & 1.0483 & 0.0351 & 0.9742 \\
\hline 5 & 5 & 0.6 & 2 & 0.5 & 2 & 30 & 1 & 0.1 & 0.5 & 0.71 & 0.5 & 1 & 1.0473 & 0.0370 & 0.9595 \\
\hline 5 & 5 & 0.6 & 2 & 0.5 & 0 & 30 & 1 & 0.1 & 0.5 & 0.71 & 0.5 & 1 & 1.0130 & 0.7089 & 0.0717 \\
\hline 5 & 5 & 0.6 & 2 & 0.5 & 0.5 & 30 & 1 & 0.1 & 0.5 & 0.71 & 0.5 & 1 & 1.0013 & 0.3914 & 0.3564 \\
\hline 5 & 5 & 0.6 & 2 & 0.5 & 1 & 30 & 1 & 0.1 & 0.5 & 0.71 & 0.5 & 1 & 1.0126 & 0.0764 & 0.7221 \\
\hline 5 & 5 & 0.6 & 2 & 0.5 & 1.5 & 30 & 1 & 0.1 & 0.5 & 0.71 & 0.5 & 1 & 1.0389 & 0.0471 & 0.8836 \\
\hline 5 & 5 & 0.6 & 2 & 0.5 & 2 & 30 & 1 & 0.1 & 0.5 & 0.71 & 0.5 & 1 & 1.0473 & 0.0370 & 0.9595 \\
\hline 5 & 5 & 0.6 & 2 & 0.5 & 2 & 10 & 1 & 0.1 & 0.5 & 0.71 & 0.5 & 1 & 1.0473 & 0.0370 & 0.9595 \\
\hline 5 & 5 & 0.6 & 2 & 0.5 & 2 & 20 & 1 & 0.1 & 0.5 & 0.71 & 0.5 & 1 & 1.0473 & 0.0370 & 0.9595 \\
\hline 5 & 5 & 0.6 & 2 & 0.5 & 2 & 30 & 1 & 0.1 & 0.5 & 0.71 & 0.5 & 1 & 1.0473 & 0.0370 & 0.9595 \\
\hline 5 & 5 & 0.6 & 2 & 0.5 & 2 & 40 & 1 & 0.1 & 0.5 & 0.71 & 0.5 & 1 & 1.0473 & 0.0370 & 0.9595 \\
\hline 5 & 5 & 0.6 & 2 & 0.5 & 2 & 50 & 1 & 0.1 & 0.5 & 0.71 & 0.5 & 1 & 1.0473 & 0.0370 & 0.9595 \\
\hline 5 & 5 & 0.6 & 2 & 0.5 & 2 & 30 & 0.2 & 0.1 & 0.5 & 0.71 & 0.5 & 1 & -0.0677 & 0.0074 & 0.1919 \\
\hline 5 & 5 & 0.6 & 2 & 0.5 & 2 & 30 & 0.4 & 0.1 & 0.5 & 0.71 & 0.5 & 1 & 0.0032 & 0.0148 & 0.3838 \\
\hline 5 & 5 & 0.6 & 2 & 0.5 & 2 & 30 & 0.6 & 0.1 & 0.5 & 0.71 & 0. & 1 & 0.2126 & 0.0222 & 0.5757 \\
\hline 5 & 5 & 0.6 & 2 & 0.5 & 2 & 30 & 0.8 & 0.1 & 0.5 & 0.71 & 0. & 1 & 506 & 296 & 7676 \\
\hline 5 & 5 & 0.6 & 2 & 0.5 & 2 & 30 & 1 & 0.1 & 0.5 & 0.71 & 0.5 & 1 & 1.0473 & 0.0370 & 0.9595 \\
\hline 5 & 5 & 0.6 & 2 & 0. & 2 & 30 & 1 & 0.1 & 0.5 & 0.71 & 0 & 1 & 0473 & & 595 \\
\hline 5 & 5 & 0.6 & 2 & 0.5 & 2 & 30 & 1 & 0.1 & 0.5 & 0.71 & 0.5 & 1 & 1.0447 & 0.0738 & 0.8922 \\
\hline 5 & 5 & 0.6 & 2 & 0.5 & 2 & 30 & 1 & 0.1 & 0.5 & 0.71 & 0.5 & 1 & 1.0468 & 0.1103 & \\
\hline 5 & 5 & 0.6 & 2 & 0.5 & 2 & 30 & 1 & 0.2 & 0.5 & 0.71 & 0.5 & 1 & 1.0527 & 0.1464 & 7971 \\
\hline 5 & 5 & 0.6 & 2 & 0.5 & 2 & 30 & 1 & 0.3 & 0.5 & 0.71 & $\Omega$ & 1 & 1.0613 & 0.1818 & 0.7639 \\
\hline 5 & 5 & 0.6 & 2 & 0 & 2 & 30 & 1 & 0.4 & 0.5 & 0.71 & 0 & 1 & 1.0473 & 0.0370 & 95 \\
\hline 5 & 5 & 0.6 & 2 & 0 & 2 & 30 & 1 & 0.5 & 1 & 0.71 & 0. & 1 & 0.6200 & 0.0370 & 595 \\
\hline 5 & 5 & 0.6 & 2 & 0 & 2 & 30 & 1 & 0.1 & 1.5 & 0.71 & 0. & 1 & 0.4448 & 0.0370 & 595 \\
\hline 5 & 5 & 0.6 & 2 & 0. & 2 & 30 & 1 & 0.1 & 2 & 0.71 & 0.5 & 1 & 0.3479 & 0.0370 & 595 \\
\hline 5 & 5 & 0.6 & 2 & 0.5 & 2 & 30 & 1 & 0.1 & 2.5 & 0.71 & 0.5 & 1 & 0.2860 & 0.0370 & 0.9595 \\
\hline 5 & 5 & 0.6 & 2 & 0.5 & 2 & 30 & 1 & 0.1 & 0.5 & 1 & 0. & 1 & 1.0488 & 0.0408 & 1.0085 \\
\hline 5 & 5 & 0.6 & 2 & 0.5 & 2 & 30 & 1 & 0.1 & 0.5 & 2 & 0. & 1 & 1.0386 & 0.0534 & 1.0594 \\
\hline 5 & 5 & 0.6 & 2 & 0. & 2 & 30 & 1 & 0.1 & 0.5 & 3 & 0. & 1 & 1.0294 & 0.0639 & 1.0700 \\
\hline 5 & 5 & 0.6 & 2 & 0. & 2 & 30 & 1 & 0.1 & 0.5 & 4 & 0. & 1 & 1.0230 & 0.0730 & 1.0714 \\
\hline 5 & 5 & 0.6 & 2 & 0.5 & 2 & 30 & 1 & 0.1 & 0.5 & 5 & 0.5 & 1 & 1.0183 & 0.0811 & 1.0694 \\
\hline 5 & 5 & 0.6 & 2 & 0.5 & 2 & 30 & 1 & 0.1 & 0.5 & 0.71 & 0 & 1 & 0.9069 & 0.0370 & 0.2752 \\
\hline 5 & 5 & 0.6 & 2 & 0. & 2 & 30 & 1 & 0.1 & 0.5 & 0.71 & 0. & 1 & 1.0473 & 0.0370 & 0.9595 \\
\hline 5 & 5 & 0.6 & 2 & 0.5 & 2 & 30 & 1 & 0.1 & 0.5 & 0.71 & 1 & 1 & 1.1019 & 0.0370 & 1.2655 \\
\hline 5 & 5 & 0.6 & 2 & 0.5 & 2 & 30 & 1 & 0.1 & 0.5 & 0.71 & 1.5 & 1 & 1.1320 & 0.0370 & 1.4627 \\
\hline 5 & 5 & 0.6 & 2 & 0.5 & 2 & 30 & 1 & 0.1 & 0.5 & 0.71 & 2 & 1 & 1.1518 & 0.0370 & 1.6124 \\
\hline 5 & 5 & 0.6 & 2 & 0.5 & 2 & 30 & 1 & 0.1 & 0.5 & 0.71 & 0.5 & 5 & 2.1569 & 0.0370 & 0.9595 \\
\hline 5 & 5 & 0.6 & 2 & 0.5 & 2 & 30 & 1 & 0.1 & 0.5 & 0.71 & 0.5 & 10 & 3.0755 & 0.0370 & 0.9595 \\
\hline 5 & 5 & 0.6 & 2 & 0.5 & 2 & 30 & 1 & 0.1 & 0.5 & 0.71 & 0.5 & 15 & 3.7897 & 0.0370 & 0.9595 \\
\hline 5 & 5 & 0.6 & 2 & 0.5 & 2 & 30 & 1 & 0.1 & 0.5 & 0.71 & 0.5 & 20 & 4.3934 & 0.0370 & 0.9595 \\
\hline 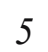 & 5 & 0.6 & 2 & 0.5 & 2 & 30 & 1 & 0.1 & 0.5 & 0.71 & 0.5 & 25 & 4.9255 & 0.0370 & 0.9595 \\
\hline
\end{tabular}




\section{Numerical results of the skin friction, Nusselt number, Sherwood number at the boundary surface are illustrated in Table}

At the plate $(y=0)$ when the Grashof number $(\mathrm{Gr})$ and modified Grashof number $(\mathrm{Gm})$ increase, the skin friction $(\tau)$ is decreasing, but no variation in the Nusselt number $(\mathrm{Nu})$ and Sherwood number is observed. The rate of skin friction $(\tau)$ decreases for increasing values of the Soret number $(\mathrm{Sr})$, thermal radiation $(R)$, permeability parameter $(K)$ and Prandtl number $(\operatorname{Pr})$, and the rate of heat transfer at the plate increases for increasing values of thermal radiation $(R)$,angle of inclination $(\alpha)$, frequency of excitation $(\omega)$ and Prandtl number (Pr), but a reverse trend is observed for increasing values of the heat source parameter $(Q)$, increasing values of the Schmidt number $(\mathrm{Sc})$, angle of inclination $(\alpha)$, chemical reaction parameter (Kr) and Prandtl number (Pr).

\section{Conclusions}

Then non-Newtonian property of heat and mass transfer effect on an MHD flow past an inclined porous plate in the occurrence of chemical reaction is studied. The results of our research study can be summarized as follows:

- The fluid velocity increases when the Grashof number (Gr), modified Grashof number $(\mathrm{Gm})$, the Soret number (Sr) increase.

- The fluid velocity decreases with an increasing radiation of parameter $(R)$, heat source parameter $(Q)$, chemical reaction parameter $(K r)$, Schmidt number $(\mathrm{Sc})$, Prandtl number (Pr) and the angle of inclination parameter $(\alpha)$.

- The fluid temperature decreases with the effect of the Prandtl number $(\operatorname{Pr})$, radiation parameter $(R)$ and heat source parameter $(Q)$.

- The concentration level of the fluid increases when the Soret number $(\mathrm{Sr})$ increases, but a reverse trend is observed for an increasing chemical reaction parameter $(\mathrm{Kr})$ and the Schmidt number $(\mathrm{Sc})$.

- The skin friction $(\tau)$ increases with an increasing rate of the chemical reaction $(\mathrm{Kr})$, magnetic field $(M)$, angle of inclination $(\alpha)$, radiation, the Schmidt number (Sc) and the Prandtl number (Pr) whereas an increase in the thermal buoyancy parameter, mass buoyancy parameter, Soret number $(\mathrm{Sr})$ and the permeability parameter $(K)$ decrease the skin friction.

- The rate of heat transfer at the wall increases with the radiation $(R)$ and Prandtl number $(\operatorname{Pr})$ and a reverse trend is observed under the influence of the heat source parameter $(Q)$.

- The rate of mass transfer at the wall enhances under the effect of the chemical reaction parameter $(\mathrm{Kr})$, Schmidt number $(\mathrm{Sc})$, heat source parameter $(Q)$ and the Sherwood number $(\mathrm{Sh})$ decreases with an increase in the thermal radiation and diffusion.

10. Appendix

$$
\begin{aligned}
& k_{1}=\sqrt{R-(Q-i \omega) \operatorname{Pr}}, \quad k_{2}=\sqrt{(K r+i \omega) \mathrm{Sc}}, \quad k_{3}=\sqrt{M+\frac{1}{K}+i \omega}, \\
& A_{1}=\frac{-\mathrm{SrSc} k_{1}^{2} t}{k_{1}^{2}-k_{2}^{2}}, \quad A_{2}=\left(t-A_{1}\right), \quad A_{3}=\frac{-\mathrm{Gr} \cos \alpha t}{k_{1}^{2}-k_{3}^{2}}, \quad A_{4}=\frac{-\mathrm{Gm} \cos \alpha A_{2}}{k_{2}^{2}-k_{3}^{2}}, \\
& A_{5}=\frac{-\mathrm{Gm} \cos \alpha A_{1}}{k_{1}^{2}-k_{3}^{2}}, \quad A_{6}=A_{3}+A_{5}, \quad A_{7}=t^{2}-\left(A_{6}+A_{4}\right) .
\end{aligned}
$$




\section{Nomenclature}

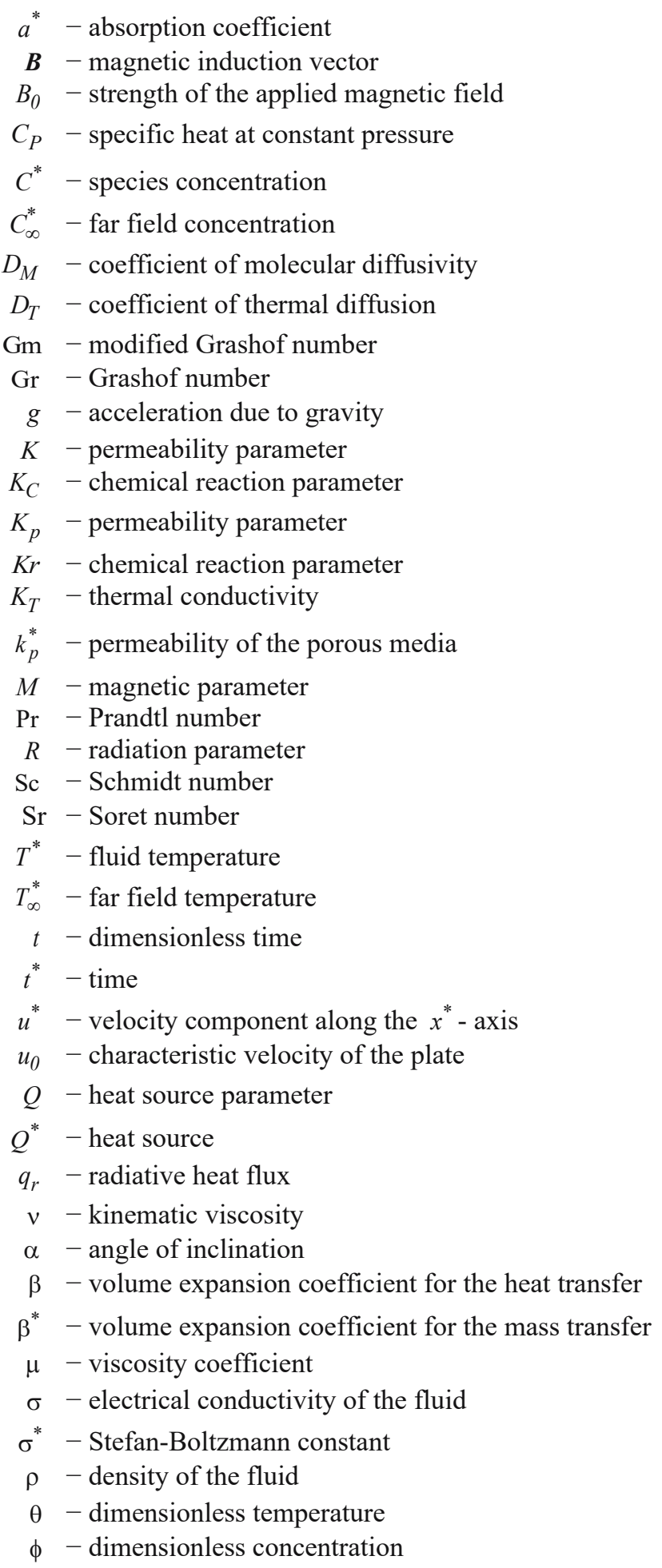

\section{References}

[1]. Fujii T. and Imura H. (1972): Natural convection heat transfer from a plate with arbitrary inclination.- Int. J. Heat Mass Tran., vol.15, pp.755-767. 
[2]. YuW.-S. and Lin H.-T. (1988): Free convection heat transfer from an isothermal plate with arbitrary inclination.- Wrme- und Stoffbertragung, vol.23, No.4, pp.203-211.

[3]. Helmy K.A. (1998): MHD unsteady free convection flow past a vertical porous plate.- Z. Angew Math. Mech., vol.78, pp.255-270.

[4]. Makinde O.D. and Mhone P.Y. (2005): Heat transfer to MHD oscillatory flow in a channel filled with porous medium.- Rom. J. Phys., vol.50, No.9-10, pp.931-938.

[5]. Bhuvaneswari M., Sivasankaran S. and Kim Y.J. (2010): Exact analysis of radiation convective flow heat and mass transfer over an inclined plate in a porous medium.- World Appl. Sci. J.. vol.10, pp.774-778.

[6]. Nayak M.K. (2016): Chemical reaction effect on MHD viscoelastic fluid over a stretching sheet through porous medium.- Meccanica, vol.51, No.8, pp.1699-1711.

[7]. Venkata Ramana Reddy G., Ramana Murthy Ch.V. and Bhaskar Reddy N. (2010): Effect of critical parameters on MHD flow in a porous channel when the suction decreases exponentially.- International Journal of Emerging Technologies and Engineering Technology and Sciences,vol.3, No.2, pp.135-139.

[8]. Gebhart B. and Pera L. (1971):The nature of vertical natural convection flows resulting from the combined buoyancy effects of thermal and mass diffusion.- Int. J. Heat and Mass Transfer, vol.14, pp.2025-2050.

[9]. Deka R.K. and Neog B.C. (2009): Combined effects of thermal radiation and chemical reaction on free convection flow past a vertical plate in porous medium.- Advances and Applications in Fluid Mechanics, vol.6, pp.181-195.

[10]. Sri Hari BabuV. and Ramana Reddy G.V. (2011): Mass transfer effects on MHD mixed convective flow from a vertical surface with Ohmic heating and viscous dissipation.- Advances in Applied Science Research, vol.2, No.4, pp.138-146.

[11]. Barik R.N., Dash G.C. and Rath P.K. (2014): Thermal radiation effect on an unsteady MHD flow past inclined porous heated plate in the presence of chemical reaction and viscous-dissipation.- Appl. Math. Comput., vol.226, No.1, pp.423-434.

[12]. Siva Reddy Sheri and Srinivasa Raju R. (2015): Soret effect on unsteady MHD free convective flow past a semiinfinite vertical plate in the presence viscous dissipation.- Int. J. Comput. Methods Eng. Sci. Mech., vol.16, pp.132-141.

[13]. Srinivasa Raju R., Jithender Reddy G., Anand Rao J., Rashidi M.M. and Gorla R.S.R. (2016): Analytical and Numerical study of unsteady MHD free convection flow over an exponentially moving vertical plate with heat absorption.- Int. J. Thermal Sci., vol.107, pp.303-315.

[14]. Mohamed Abd El-Aziz and Aishah S. Yahya (2017): Heat and mass transfer of unsteady hydromagnetic free convection flow through porous medium past a vertical plate with uniform surface heat flux.- Journal of Theoretical and Applied Mechanics, Sofia, vol.47, No.3, pp.25-58.

[15]. Ramana Reddy G.V., Bhaskar Reddy N. and Gorla R.S.R. (2016): Radiation and chemical reaction effects on MHD flow along a moving vertical porous plate.- International Journal of Applied Mechanics and Engineering.

[16]. Mangathai P., Ramana Reddy G.V. and Rami Reddy B. (2016):MHD free convective flow past a vertical porous plate in the presence of radiation and heat generation.- International Journal of Chemical Sciences.

[17]. Sreedevi G., Prasada Rao D.R.V., Makinde O.D. and Venkata Ramana Reddy G. (): Soret and Dufour effects on MHD flow with heat and mass transfer past a permeable stretching sheet in presence of thermal radiation.Indian Journal of Pure and Applied Physics.

[18]. Reddy G.V.R., Ibrahim S.M. and Bhagavan V.S. (2014): Similarity transformations of heat and mass transfer effects on steady MHD free convection dissipative fluid flow past an inclined porous surface with chemical reaction.- Journal of Naval Architecture and Marine Engineering. 